\title{
Effects of Preoperative Neutrophil-to-Lymphocyte and Platelet-to-Lymphocyte Ratios on Survival in Patients with Extrahepatic Cholangiocarcinoma
}

\author{
YUKI KITANO, YO-ICHI YAMASHITA, KENSUKE YAMAMURA, KOTA ARIMA, \\ TAKAYOSHI KAIDA, TATSUNORI MIYATA, SHIGEKI NAKAGAWA, KOSUKE MIMA, \\ KATSUNORI IMAI, DAISUKE HASHIMOTO, AKIRA CHIKAMOTO and HIDEO BABA \\ Department of Gastroenterological Surgery, Graduate School of Medical Sciences, \\ Kumamoto University, Kumamoto, Japan
}

\begin{abstract}
Background/Aim: As indicators of systemic inflammatory response, the neutrophil-to-lymphocyte ratio $(N L R)$ and platelet-to-lymphocyte ratio (PLR) predict prognoses for various cancers. This study investigated their prognostic significance in extrahepatic cholangiocarcinoma (ECC). Patients and Methods: We analyzed 120 patients who underwent surgery for ECC between 2000 and 2014. We calculated preoperative NLR and PLR and evaluated their correlations with patients' clinicopathological features and prognosis. Results: Although high NLR was not associated with worse recurrence-free survival (RFS) (hazard ratio $(H R)=1.32$, $p=0.26)$, cancer-specific survival $(C S S)(H R=1.35, p=0.31)$ and overall survival $(O S)(H R=1.19, p=0.52)$, high PLR was significantly associated with worse RFS $(H R=1.85, p=0.01)$, CSS $(H R=2.38, p=0.002)$ and $O S(H R=1.98, p=0.008)$. In multivariate analysis, high PLR $(H R=1.89, p=0.02)$ and lymph node metastasis $(H R=1.78, p=0.03)$ were independent prognostic factors for OS. A high PLR had more liver recurrences $(p=0.04)$ and recurrences within 1 year $(H R=2.38$, $p=0.02)$ than low PLR. Conclusion: High preoperative PLR was an independent predictor of poor prognosis for patients with ECC who underwent resections.
\end{abstract}

Extrahepatic cholangiocarcinoma (ECC), including perihilar cholangiocarcinoma and distal cholangiocarcinoma, is a devastating disease that presents challenges for hepatobiliary

Correspondence to: Hideo Baba, MD, Ph.D., FACS, Department of Gastroenterological Surgery, Graduate School of Medicine, Kumamoto University, 1-1-1 Honjo, Chuo-ku, Kumamoto 8608556, Japan. Tel: +81 963735211, Fax: +81 963714378, e-mail: hdobaba@kumamoto-u.ac.jp

Key Words: Extrahepatic cholangiocarcinoma, neutrophil-tolymphocyte ratio, platelet-to-lymphocyte ratio. surgeons (1-4). Complete resection of these tumors remains the only curative treatment (1). Although surgical techniques, perioperative management and postoperative treatments have advanced and the R0 resection rate has increased, patients' prognoses remain unsatisfactory; therefore additional treatment strategies should be developed (4-6).

The ability to predict prognosis preoperatively is critical for selection of treatment plan and follow-up strategies. Recently, systemic inflammatory responses, such as C-reactive protein (CRP), albumin, Glasgow prognostic score (GPS), neutrophilto-lymphocyte ratio (NLR) and platelet-lymphocyte ratio (PLR), have been found to be independent prognostic markers for various solid cancers (7-11). Supporting evidence suggests that the systemic inflammatory response affects tumor growth and metastasis (12-14). However, the prognostic significance of these markers in patients with ECC has not been studied.

Therefore, this study investigated whether NLR and PLR are correlated with the prognosis of patients with ECC who undergo surgical resection.

\section{Patients and Methods}

Patients. We enrolled 120 patients with ECC who underwent surgical resections at the Kumamoto University Hospital, Kumamoto, Japan, between 2000 and 2014. All patients had pathologically confirmed diagnoses of cholangiocarcinoma. All patients gave written informed consent and the Ethics Committee of Kumamoto University approved the study's protocol. Pathological findings were prospectively documented according to the Japanese standardized reporting format for biliary tract cancers (15) and the pathological tumor (T), node $(\mathrm{N})$ and metastasis $(\mathrm{M})$ classifications were re-labeled according to the American Joint Committee on Cancer (AJCC) system, seventh edition (16).

Blood samples. Blood samples were obtained preoperatively. In patients who had obstructive jaundice, blood samples were obtained after total bilirubin levels decreased to $<2.0 \mathrm{mg} / \mathrm{dl}$ by treatment to reduce jaundice. 
Evaluation and statistical analysis. NLR was defined as neutrophil count $(/ \mu \mathrm{l})$ divided by lymphocyte count $(/ \mu \mathrm{l})$; PLR was defined as platelet count $(/ \mu \mathrm{l})$ divided by the lymphocyte count $(/ \mu \mathrm{l})$. We identified a cut-off value for continuous value of NLR and PLR as third quartile. GPS was defined by the following criteria: (i) patients with both elevated CRP $(>1.0 \mathrm{mg} / \mathrm{dl})$ and hypoalbuminemia $(<3.5$ $\mathrm{g} / \mathrm{dl}$ ) were allocated a score of 2; (ii) patients with only one of these biochemical abnormalities were allocated a score of 1 ; and (iii) patients in whom neither of these abnormalities was present were allocated a score of 0 (8). Continuous variables were expressed as means \pm standard deviation; differences were assessed for significance using Student's $t$-test or the Mann-Whitney test. Categorical variables were evaluated using Chi-square or Fisher's exact tests, as appropriate. Cox proportional hazard regression analyses were performed to identify predictors of prognosis. Recurrence-free survival (RFS), cancer-specific survival (CSS) and overall survival (OS) rates were estimated using the Kaplan-Meier method, while survival curves were compared using the log-rank test. Pearson's correlation methods were performed to identify correlations for quantitative variables following normal distribution. $p<0.05$ was considered significant. All tests were performed on JMP software version 10.0.2 (SAS Institute Inc., Cary, NC, USA).

\section{Results}

Clinicopathological characteristics and perioperative outcome of patients. Patients' clinicopathologic characteristics and their perioperative outcomes categorized by high/low NLR and PLR are summarized in Table I. The cut-off values were determined by third quartile categorical variable to be $\mathrm{NLR}=2.8$; and $\mathrm{PLR}=185$. Of 120 patients, 57 patients $(47.5 \%)$ had perihilar ECC and 63 patients $(52.5 \%)$ distal ECC. Surgical procedures were pancreaticoduodenectomy, 59 patients (49.2\%); hemihepatectomy, 40 patients $(33.3 \%)$; extrahepatic bile duct resections only, 15 patients $(12.5 \%)$; trisectionectomy, 4 patients (3.3\%); and hepato-pancreaticoduodenectomy, 2 patients $(1.7 \%)$. For all patients, mean values of preoperative blood markers were neutrophils, $3.30 \times 10^{3} / \mu \mathrm{l}$; lymphocytes, $1.59 \times 10^{3} / \mu \mathrm{l}$; platelet counts, $22.2 \times 10^{4} / \mu \mathrm{l}$; NLR=2.4; and PLR=153.

The high NLR group had a significantly higher white blood cell (WBC) level $(p=0.04)$ and carbohydrate antigen (CA)19-9 level ( $p=0.04)$ than the low NLR group; whereas the high PLR group had significantly lower albumin level $(p=0.01)$, higher CRP level $(p=0.02)$ and poorer histologic grades $(p=0.008)$ than the low PLR group.

Relationships between long-term outcomes and NLR/PLR. The high and low NLR groups did not significantly differ in RFS (5-year RFS=8.7\% vs. $33.1 \%, \mathrm{HR}=1.32, p=0.26$; Figure 1a), CSS (5-year CSS $=24.0 \%$ vs. $42.5 \%, \mathrm{HR}=1.35, p=0.31$; Figure 1 b) or OS (5-year OS=21.8\% vs. $36.9 \%, \mathrm{HR}=1.19$, $p=0.52$; Figure $1 \mathrm{c})$. In contrast, the high and low PLR groups significantly differ in RFS (5-year RFS $=4.7 \%$ vs. $35.5 \%$, $\mathrm{HR}=1.85, p=0.01$; Figure 2a), CSS (5-year CSS $=15.5 \%$ vs. $47.4 \%, \mathrm{HR}=2.38, p=0.002$; Figure 2b) and OS (5-year $\mathrm{OS}=14.5 \%$ vs. $40.7 \%, \mathrm{HR}=1.98, p=0.008$; Figure $2 \mathrm{c}$ ). Thus,
PLR was a more accurate predictor of outcome in patients with ECC who underwent surgical resection than NLR.

Univariate and multivariate analysis for RFS, CSS and OS. Univariate and multivariable analyses of RFS, CSS and OS are summarized in Tables II, III and IV, respectively. Univariate analysis for RFS revealed that high PLR, lymph node metastasis and $\mathrm{R} 1$ resection were significantly associated with poor RFS rates; multivariate analysis of these factors showed high PLR $(\mathrm{HR}=1.81, p=0.02)$ and lymph node metastasis $(\mathrm{HR}=1.77$, $p=0.02$ ) to be independent predictors of poor RFS rates (Table II). Univariate analysis for OS revealed that high PLR, lymph node metastasis and $\mathrm{R} 1$ resection were significantly associated with poor OS rates; multivariate analysis of these factors showed high PLR $(\mathrm{HR}=1.89, p=0.02)$ and lymph node metastasis $(\mathrm{HR}=1.78, p=0.03)$ to be independent predictors of poor OS rates (Table IV). As is the case with RFS and OS, high PLR (HR=2.29, $p=0.006)$ and lymph node metastasis $(\mathrm{HR}=2.28, p=0.004)$ were independent predictors of poor CSS rates (Table III).

Relationship between recurrent sites, early recurrence and NLR/PLR. Relationships between recurrence sites, early recurrences and NLR/PLR are summarized in Table $\mathrm{V}$ and Figure 3, respectively. Although numbers of patients with recurrences and NLR/PLR were not significantly related, the high PLR group had liver recurrences more frequently than the low PLR group ( $p=0.04)$ and early recurrences within 1 year more frequently than the low PLR group $(\mathrm{HR}=2.38$, $p=0.02$; Figure 3).

Relationship between other markers of inflammation and $N L R / P L R$. Relationships between other systemic inflammatory markers and NLR/PLR are summarized in Figure 4. Although PLR correlated with other systemic inflammatory markers, such as albumin $(\mathrm{r}=-0.28, p=0.002)$ and CRP ( $\mathrm{r}=0.19, p=0.03)$, as well as GPS scores $(p=0.01)$ (8), NLR did not correlate with these factors. These results indicate that PLR reflects systemic inflammation more accurately that does NLR in patients with ECC.

\section{Discussion}

In this study, we found that high preoperative PLR, but not NLR, was an independent predictor of poor prognosis in patients with ECC who underwent surgical resection. In addition, we found that the high PLR group had more liver recurrence and early recurrence within 1 year; and that PLR, but not NLR, was correlated with other systemic inflammatory markers. Although some studies have compared NLR with PLR in relation to outcomes for patients who underwent surgeries for various gastrointestinal cancers (17-19), this is the first study that compared NLR with PLR in patients with ECC. Interestingly, our study revealed that only high PLR was an independent predictor of poor prognosis in RFS, CSS and OS. 
Kitano et al: NLR and PLR in Extrahepatic Cholangiocarcinoma

Table I. Comparisons of clinical characteristics and perioperative outcome of patients.

\begin{tabular}{|c|c|c|c|c|c|c|}
\hline & \multicolumn{3}{|c|}{ NLR } & \multicolumn{3}{|c|}{ PLR } \\
\hline & $\begin{array}{l}\text { High } \\
(\mathrm{n}=30)\end{array}$ & $\begin{array}{c}\text { Low } \\
(\mathrm{n}=90)\end{array}$ & $p$-Value & $\begin{array}{l}\text { High } \\
(\mathrm{n}=30)\end{array}$ & $\begin{array}{l}\text { Low } \\
(\mathrm{n}=90)\end{array}$ & $p$-Value \\
\hline Age (years) & $67.2 \pm 12.7$ & $67.9 \pm 9.0$ & 0.73 & $67.6 \pm 10.0$ & $67.8 \pm 9.7$ & 0.91 \\
\hline Gender & & & 0.82 & & & 0.26 \\
\hline Male & $20(66.7 \%)$ & $62(68.9 \%)$ & & $18(60.0 \%)$ & $64(71.1 \%)$ & \\
\hline Female & $10(33.3 \%)$ & $28(31.1 \%)$ & & $12(40.0 \%)$ & $26(28.9 \%)$ & \\
\hline Tumor location & & & 0.92 & & & 0.60 \\
\hline Perihilar & $14(46.7 \%)$ & $43(47.8 \%)$ & & $13(43.3 \%)$ & $44(48.9 \%)$ & \\
\hline Distal & $16(53.3 \%)$ & $47(52.2 \%)$ & & $17(56.7 \%)$ & $46(51.1 \%)$ & \\
\hline DM & & & 0.34 & & & 0.63 \\
\hline Present & $6(20.0 \%)$ & $26(28.9 \%)$ & & $9(30.0 \%)$ & $23(25.6 \%)$ & \\
\hline Absent & $24(80.0 \%)$ & $64(71.1 \%)$ & & $21(70.0 \%)$ & $67(74.4 \%)$ & \\
\hline BMI & $22.8 \pm 3.2$ & $23.6 \pm 3.5$ & 0.25 & $23.2 \pm 4.0$ & $23.5 \pm 3.3$ & 0.72 \\
\hline Reduction of jaundice & & & 0.90 & & & 0.72 \\
\hline Present & $22(73.3 \%)$ & $67(74.4 \%)$ & & $23(76.7 \%)$ & $66(73.3 \%)$ & \\
\hline Absent & $8(26.7 \%)$ & $23(25.6 \%)$ & & $7(23.3 \%)$ & $24(26.7 \%)$ & \\
\hline Albumin, $\mathrm{g} / \mathrm{dl}$ & $3.7 \pm 0.5$ & $3.8 \pm 0.4$ & 0.28 & $3.6 \pm 0.4$ & $3.8 \pm 0.4$ & 0.01 \\
\hline Bilirubin $\mathrm{mg} / \mathrm{dl}$ (1st visit) & $5.8 \pm 6.1$ & $6.6 \pm 6.5$ & 0.60 & $5.3 \pm 5.9$ & $6.7 \pm 6.5$ & 0.28 \\
\hline $\mathrm{CRP}, \mathrm{mg} / \mathrm{dl}$ & $0.9 \pm 1.1$ & $0.6 \pm 0.9$ & 0.14 & $1.0 \pm 1.3$ & $0.5 \pm 0.8$ & 0.02 \\
\hline $\mathrm{WBC} / \mu \mathrm{l}$ & $6,070 \pm 1,569$ & $5,362 \pm 1,625$ & 0.04 & $5,077 \pm 1,484$ & $5,693 \pm 1,660$ & 0.07 \\
\hline Neutrophils/ $\mu \mathrm{l}$ & $4,397 \pm 1,274$ & $2,975 \pm 1,152$ & $<0.0001$ & $3,385 \pm 1,282$ & $3,312 \pm 1,353$ & 0.8 \\
\hline 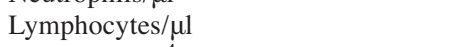 & $1,100 \pm 343$ & $1,770 \pm 623$ & $<0.0001$ & $1,127 \pm 477$ & $1,762 \pm 604$ & $<0.0001$ \\
\hline Platelets, $\times 10^{4} / \mu \mathrm{l}$ & $21.3 \pm 6.0$ & $22.4 \pm 8.2$ & 0.52 & $27.0 \pm 11.0$ & $20.5 \pm 5.4$ & $<0.0001$ \\
\hline CEA, ng/ml & $2.7 \pm 2.1$ & $2.9 \pm 8.7$ & 0.88 & $2.4 \pm 2.2$ & $3.0 \pm 8.7$ & 0.68 \\
\hline CA19-9, U/ml & $694.4 \pm 1791$ & $206.8 \pm 804.4$ & 0.04 & $656.7 \pm 1668$ & $219.4 \pm 893.0$ & 0.07 \\
\hline Operation time (min) & $681.1 \pm 291$ & $664.0 \pm 182$ & 0.70 & $696.5 \pm 292$ & $658.8 \pm 181$ & 0.40 \\
\hline Blood loss (ml) & $1,215 \pm 745$ & $1,422 \pm 1,849$ & 0.56 & $1,208 \pm 706$ & $1,423 \pm 1,853$ & 0.54 \\
\hline Procedures & & & 0.56 & & & 0.13 \\
\hline Extrahepatic bile duct resection & $5(16.7 \%)$ & $10(11.1 \%)$ & & $2(6.7 \%)$ & $13(14.4 \%)$ & \\
\hline Hemihepatectomy & $9(30.0 \%)$ & $31(34.4 \%)$ & & $10(33.3 \%)$ & $30(33.3 \%)$ & \\
\hline Trisectionectomy & $2(6.7 \%)$ & $2(2.2 \%)$ & & $3(10.0 \%)$ & $1(1.1 \%)$ & \\
\hline Pancreaticoduodenectomy & $13(43.3 \%)$ & $46(51.1 \%)$ & & $15(50.0 \%)$ & $44(48.9 \%)$ & \\
\hline Hepato-pancreaticoduodenectomy & $1(3.3 \%)$ & $1(1.1 \%)$ & & 0 & $2(2.2 \%)$ & \\
\hline Postoperative complication ( $\mathrm{CD} \geq \mathrm{IIIa})$ & & & 0.11 & & & 0.92 \\
\hline Present & $13(43.3 \%)$ & $54(60.0 \%)$ & & $17(56.7 \%)$ & $50(55.6 \%)$ & \\
\hline Absent & $17(56.7 \%)$ & $36(40.0 \%)$ & & $13(43.3 \%)$ & $40(44.4 \%)$ & \\
\hline Deaths & & & 0.24 & & & 1 \\
\hline Present & 0 & $4(4.4 \%)$ & & $1(3.3 \%)$ & $3(3.3 \%)$ & \\
\hline Absent & $30(100 \%)$ & $86(95.6 \%)$ & & $29(96.7 \%)$ & $87(96.7 \%)$ & \\
\hline Tumor size (mm) & $31 \pm 17$ & $29 \pm 14$ & 0.57 & $30 \pm 15$ & $29 \pm 14$ & 0.83 \\
\hline Histologic grade & & & 0.60 & & & 0.008 \\
\hline Papillary & 0 & $4(4.4 \%)$ & & 0 & $4(4.4 \%)$ & \\
\hline Well & $14(46.7 \%)$ & $41(45.6 \%)$ & & $7(23.3 \%)$ & $48(53.3 \%)$ & \\
\hline Moderate & $14(46.7 \%)$ & $36(40.0 \%)$ & & $20(66.7 \%)$ & $30(33.3 \%)$ & \\
\hline Poor & $2(6.7 \%)$ & $9(10.0 \%)$ & & $3(10.0 \%)$ & $8(8.9 \%)$ & \\
\hline pT & & & 0.59 & & & 0.21 \\
\hline $\mathrm{T} 1$ & $1(3.3 \%)$ & $9(10.0 \%)$ & & 0 & $10(11.1 \%)$ & \\
\hline $\mathrm{T} 2$ & $12(40.0 \%)$ & $40(44.4 \%)$ & & $12(40.0 \%)$ & $40(44.4 \%)$ & \\
\hline $\mathrm{T} 3$ & $16(53.3 \%)$ & $39(43.3 \%)$ & & $17(56.7 \%)$ & $38(42.2 \%)$ & \\
\hline $\mathrm{T} 4$ & $1(3.3 \%)$ & $2(2.2 \%)$ & & $1(3.3 \%)$ & $2(2.2 \%)$ & \\
\hline $\mathrm{pN}$ & & & 0.51 & & & 0.83 \\
\hline N0 & $21(70.0 \%)$ & $57(63.3 \%)$ & & $19(63.3 \%)$ & $59(65.6 \%)$ & \\
\hline N1 & $9(30.0 \%)$ & $33(36.7 \%)$ & & $11(36.7 \%)$ & $31(34.4 \%)$ & \\
\hline R status & & & 0.36 & & & 0.17 \\
\hline R0 & $19(63.3 \%)$ & $65(72.2 \%)$ & & $18(60.0 \%)$ & $66(73.3 \%)$ & \\
\hline $\mathrm{R} 1$ & $11(36.7 \%)$ & $25(27.8 \%)$ & & $12(40.0 \%)$ & $24(26.7 \%)$ & \\
\hline Adjuvant therapy & & & 0.11 & & & 0.75 \\
\hline Present & $17(56.7 \%)$ & $36(40.0 \%)$ & & $14(46.7 \%)$ & $39(43.3 \%)$ & \\
\hline Absent & $13(43.3 \%)$ & $54(60.0 \%)$ & & $16(53.3 \%)$ & $51(56.7 \%)$ & \\
\hline
\end{tabular}

NLR, Neutrophil-to-lymphocyte ratio; PLR, platelet-to-lymphocyte ratio; BMI, body mass index; CA19-9, carbohydrate antigen 19-9; CEA, carcinoembryonic antigen; CRP, C-reactive protein; DM, diabetes mellitus; WBC, white blood cells; CD, Clavien-Dindo classification; pT, pathological tumor; $\mathrm{pN}$, pathological node. Data are shown as mean \pm standard deviation or $\mathrm{n}(\%)$. Bold values are significant at $p<0.05$. 
(a)

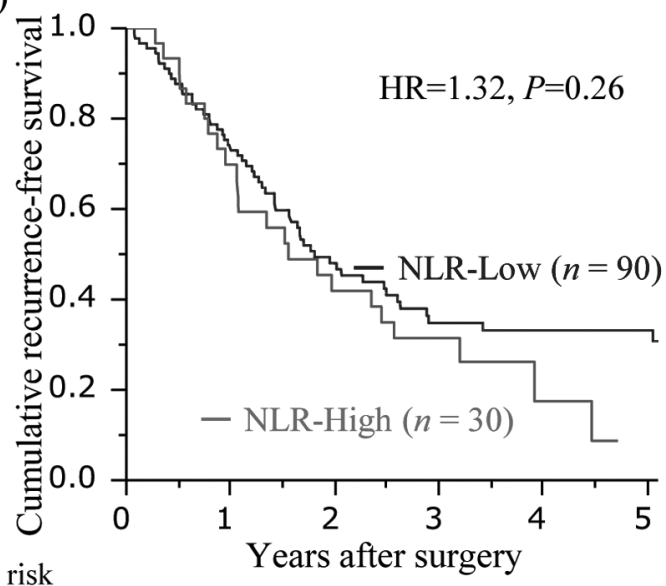

No. at risk

$\begin{array}{ccccccc}\text { NLR-Low } & 90 & 65 & 37 & 23 & 20 & 15 \\ \text { NLR-High } & 30 & 21 & 13 & 8 & 3 & 1\end{array}$

(b)

No. at risk

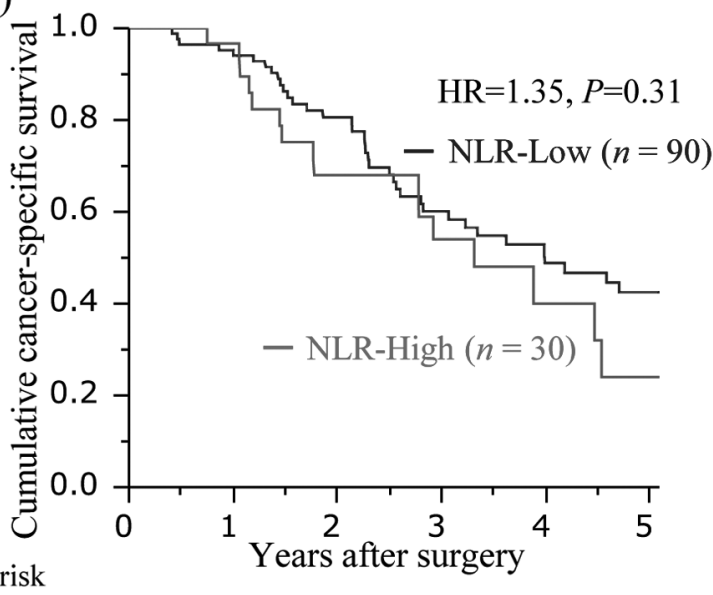

The mechanisms that associate high PLR with poor prognosis have been gradually understood. Interleukin-1 and -6 can promote megakaryocyte proliferation and differentiation of megakaryocytes to platelets (20-23). Also, platelets themselves are a critical source of growth factors, especially transforming growth factor $\beta$ (TGF $\beta$ ), vascular endothelial growth factor (VEGF), platelet-derived growth factor (PDGF) and platelet factor 4 (PF4) that can stimulate angiogenesis and promote tumor progression and hematogenous metastasis $(12,14,24,25)$, thus tying high PLR to negative prognoses in several cancers. Meanwhile, development of molecular target therapy for these factors would be desired in the future. (c)

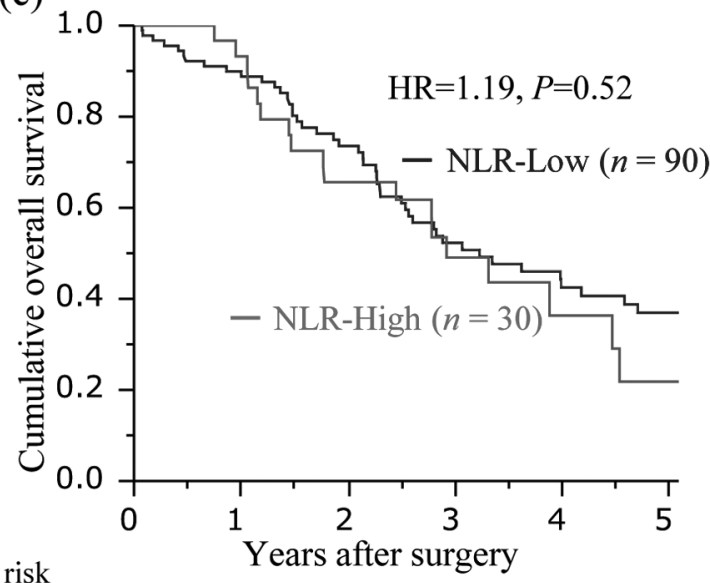

No. at risk

$\begin{array}{ccccccc}\text { NLR-Low } & 90 & 79 & 55 & 35 & 25 & 18 \\ \text { NLR-High } & 30 & 28 & 19 & 11 & 6 & 3\end{array}$

Figure 1. (a) Recurrence-free survival curves by NLR. (b) Cancerspecific survival curves by NLR. (c) Overall survival curves by NLR. $N L R$, Neutrophil-to-lymphocyte ratio; HR, hazard ratio.

Since ECC patients have poor prognoses, even if curative resections are achieved, development of accurate predictive markers has been sorely needed. In patients with ECC, lymph node metastasis $(26,27)$, poor histological differentiation $(28$, $29)$, positive surgical margins $(30,31)$ and pre-/post-CA19-9 levels $(32,33)$ have been reported to be independent prognostic factors. However, since most of these data could only be obtained postoperatively, their preoperative value in determining a patient's treatment strategy has been very limited. Preoperative PLR is a simple, inexpensive and reproducible measurement. Furthermore, although high preoperative CA19-9 (reported to be a prognostic factor in ECC) is routinely considered for these patients (32), our analysis revealed PLR to be a more accurate prognostic factor than preoperative CA19-9. Moreover, our study showed that patients with high PLR had more frequent liver recurrences and recurrences within 1 year of surgery than did patients with low PLR. These results suggest that surgeons might want to administer adjuvant chemotherapy or hepatic arterial infusion chemotherapy to patients with high PLR values $(34,35)$.

Although both NLR and PLR have been shown to be independent predictors of poor prognosis in various cancers $(9,10,36,37)$, some studies found that NLR was a more accurate predictor than PLR for gastrointestinal cancers (17, 18). This is the first study to show the superiority of PLR to NLR in predicting poor prognosis in patients who undergo surgical treatment for ECC. In our study, high PLR is 
(a)

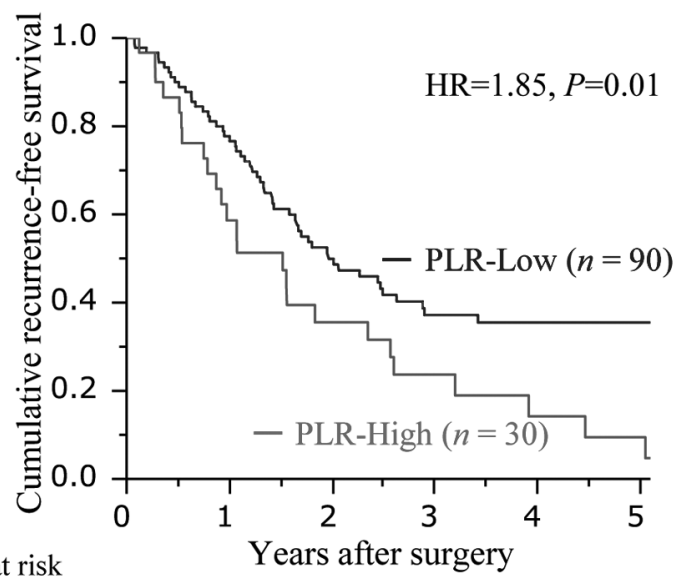

No. at risk

$\begin{array}{ccccccc}\text { PLR-Low } & 90 & 68 & 40 & 25 & 19 & 13 \\ \text { PLR-High } & 30 & 17 & 10 & 6 & 4 & 3\end{array}$

(b)

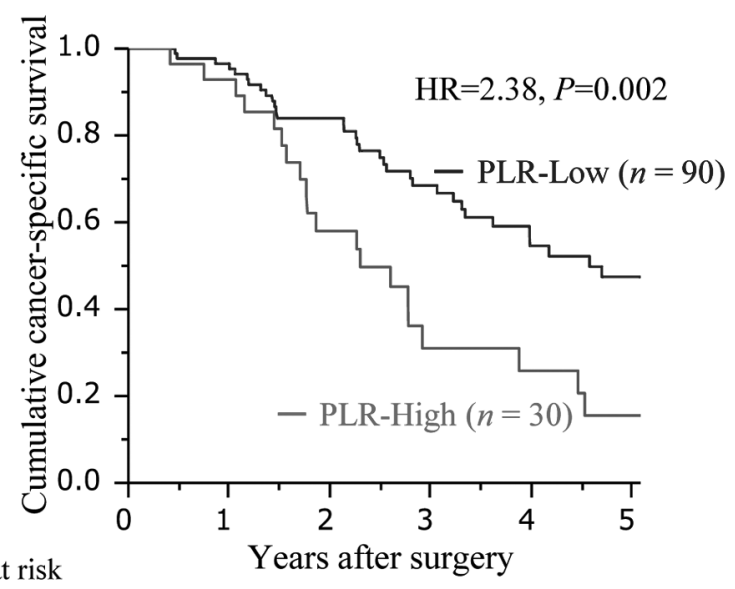

No. at risk

$\begin{array}{ccccccc}\text { PLR-Low } & 90 & 81 & 59 & 39 & 25 & 17 \\ \text { PLR-High } & 30 & 26 & 15 & 7 & 6 & 4\end{array}$

superior to high NLR as a prognostic factor probably because PLR can reflect systemic inflammation more accurately than NLR in patients with ECC. Our analysis shows that only PLR correlated with other inflammatory markers, such as CRP, albumin and GPS (Figure 4). Moreover, Shirai et al. also revealed that, in patients with pancreatic cancer who underwent surgical resection, PLR was an independent poor prognostic factor, but NLR was not, and advocated that systemic inflammatory changes by biliary drainage may improve patients' prognosis (37).

The present study has limitations associated with its retrospective design conducted at a single center, limited (c)

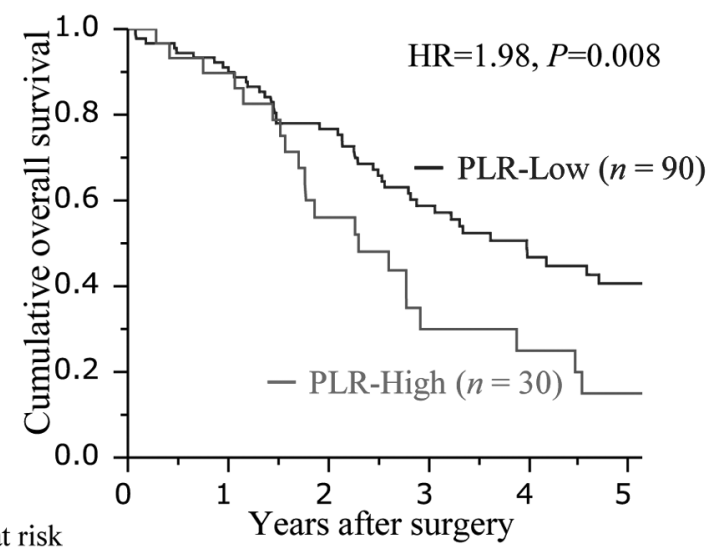

No. at risk

$\begin{array}{ccccccc}\text { PLR-Low } & 90 & 81 & 59 & 39 & 25 & 17 \\ \text { PLR-High } & 30 & 26 & 15 & 7 & 6 & 4\end{array}$

Figure 2. (a) Recurrence-free survival curves by PLR. (d) Cancerspecific survival curves by PLR. (c) Overall survival curves by PLR. $P L R$, Platelet-to-lymphocyte ratio; HR, hazard ratio.

number of patients and enrolled patients for a long duration, which might introduce several biases. Hence, a prospective multicenter study would be desirable to validate our present findings.

In conclusion, high preoperative PLR can be an independent predictor of poor prognosis in patients with ECC who undergo surgical resection. This readily-available serum biomarker may, thus, help guide additional treatment strategies.

\section{Ethical Standards}

All procedures followed were in accordance with the ethical standards of the responsible committees on human experimentation (institutional and national) and with the Helsinki Declaration of 1964 and later versions. Informed consents or substitutes for them were obtained from all patients for their inclusion in this study.

\section{Conflicts of Interest}

The Authors declare that this study had no grant support and the Authors have no conflicts of interest.

\section{References}

1 Nakeeb A, Pitt HA, Sohn TA, Coleman J, Abrams RA, Piantadosi $\mathrm{S}$ and Cameron JL: Cholangiocarcinoma. A spectrum of intrahepatic, perihilar, and distal tumors. Ann Surg 224: 463-473, 1996. 
Table II. Univariate and multivariate analyses of factors that affect recurrence-free survival in patients with extrahepatic cholangiocarcinoma.

\begin{tabular}{|c|c|c|c|c|c|c|}
\hline \multirow[b]{2}{*}{ Variables } & \multicolumn{3}{|c|}{ Univariate analysis } & \multicolumn{3}{|c|}{ Multivariate analysis } \\
\hline & HR & $95 \% \mathrm{CI}$ & $p$-Value & HR & $95 \% \mathrm{CI}$ & $p$-Value \\
\hline Age $>75$ years & 1.13 & $0.68-1.82$ & 0.63 & & & \\
\hline Male gender & 0.77 & $0.49-1.22$ & 0.26 & & & \\
\hline $\mathrm{BMI}>25.0$ & 0.84 & $0.48-1.40$ & 0.52 & & & \\
\hline Reduction of jaundice & 1.09 & $0.67-1.85$ & 0.75 & & & \\
\hline Bilirubin $>10 \mathrm{mg} / \mathrm{dl}$ (1st visit) & 1.29 & $0.76-2.09$ & 0.34 & & & \\
\hline Albumin $<4.0 \mathrm{~g} / \mathrm{dl}$ & 1.45 & $0.90-2.44$ & 0.13 & & & \\
\hline NLR $>2.8$ & 1.32 & $0.80-2.13$ & 0.27 & & & \\
\hline PLR > 185 & 1.85 & $1.13-2.95$ & 0.02 & 1.81 & $1.10-2.91$ & 0.02 \\
\hline $\mathrm{CEA}>3.0 \mathrm{ng} / \mathrm{ml}$ & 0.91 & $0.49-1.57$ & 0.74 & & & \\
\hline CA19-9 >120 U/ml & 1.46 & $0.88-2.34$ & 0.14 & & & \\
\hline Tumor size $>40 \mathrm{~mm}$ & 1.30 & $0.70-2.24$ & 0.39 & & & \\
\hline Surgical time $>720 \mathrm{~min}$ & 1.46 & $0.91-2.30$ & 0.11 & & & \\
\hline Blood loss $>1,500 \mathrm{cc}$ & 1.43 & $0.87-2.30$ & 0.15 & & & \\
\hline Postoperative complication & 1.06 & $0.68-1.67$ & 0.78 & & & \\
\hline Perihilar tumor location & 1.09 & $0.71-1.69$ & 0.69 & & & \\
\hline Poor histologic grade & 0.8 & $0.36-1.58$ & 0.55 & & & \\
\hline $\mathrm{T} 3,4$ & 1.35 & $0.86-2.11$ & 0.19 & & & \\
\hline N1 & 1.84 & $1.16-2.89$ & 0.01 & 1.77 & $1.10-2.80$ & 0.02 \\
\hline $\mathrm{R} 1$ & 1.73 & $1.06-2.75$ & $\mathbf{0 . 0 3}$ & 1.45 & $0.88-2.34$ & 0.14 \\
\hline No adjuvant therapy & 1.13 & $0.73-1.78$ & 0.58 & & & \\
\hline
\end{tabular}

BMI, Body mass index; CI, confidence interval; CA19-9, carbohydrate antigen 19-9; CEA, carcinoembryonic antigen; HR, hazard ratio; NLR, neutrophil-to-lymphocyte ratio; PLR, platelet-to-lymphocyte ratio. Bold values are significant at $p<0.05$.

Table III. Univariate and multivariate analysis of factors that affect cancer-specific survival in patients with extrahepatic cholangiocarcinoma.

\begin{tabular}{|c|c|c|c|c|c|c|}
\hline \multirow[b]{2}{*}{ Variables } & \multicolumn{3}{|c|}{ Univariate analysis } & \multicolumn{3}{|c|}{ Multivariate analysis } \\
\hline & HR & $95 \% \mathrm{CI}$ & $p$-Value & HR & $95 \% \mathrm{CI}$ & $p$-Value \\
\hline Age $>75$ years & 0.92 & $0.45-1.72$ & 0.80 & & & \\
\hline Male gender & 0.61 & $0.36-1.04$ & 0.07 & & & \\
\hline BMI $>25.0$ & 0.91 & $0.46-1.66$ & 0.76 & & & \\
\hline Reduction of jaundice & 1.16 & $0.62-2.37$ & 0.65 & & & \\
\hline Bilirubin $>10 \mathrm{mg} / \mathrm{dl}$ (1st visit) & 0.89 & $0.44-1.65$ & 0.72 & & & \\
\hline Albumin $<4.0 \mathrm{~g} / \mathrm{dl}$ & 1.66 & $0.91-3.24$ & 0.10 & & & \\
\hline NLR $>2.8$ & 1.35 & $0.73-2.39$ & 0.33 & & & \\
\hline PLR $>185$ & 2.38 & $1.34-4.12$ & 0.004 & 2.29 & $1.29-3.97$ & 0.006 \\
\hline CEA > $3.0 \mathrm{ng} / \mathrm{ml}$ & 0.97 & $0.44-1.89$ & 0.92 & & & \\
\hline CA19-9 >120 U/ml & 1.16 & $0.61-2.08$ & 0.64 & & & \\
\hline Tumor size $>40 \mathrm{~mm}$ & 0.78 & $0.32-1.65$ & 0.54 & & & \\
\hline Surgical time $>720 \mathrm{~min}$ & 1.37 & $0.77-2.34$ & 0.27 & & & \\
\hline Blood loss $>1,500 \mathrm{cc}$ & 1.54 & $0.87-2.66$ & 0.13 & & & \\
\hline Postoperative complication & 0.94 & $0.55-1.62$ & 0.82 & & & \\
\hline Perihilar tumor location & 1.07 & $0.62-1.81$ & 0.81 & & & \\
\hline Poor histologic grade & 0.81 & $0.28-1.84$ & 0.64 & & & \\
\hline $\mathrm{T} 3,4$ & 1.15 & $0.67-1.96$ & 0.62 & & & \\
\hline N1 & 2.41 & $1.41-4.14$ & 0.002 & 2.28 & $1.31-3.96$ & 0.004 \\
\hline $\mathrm{R} 1$ & 2.03 & $1.12-3.55$ & 0.02 & 1.65 & $0.90-2.92$ & 0.1 \\
\hline No adjuvant therapy & 0.87 & $0.51-1.49$ & 0.61 & & & \\
\hline
\end{tabular}

BMI, Body mass index; CI, confidence interval; CA19-9, carbohydrate antigen 19-9; CEA, carcinoembryonic antigen; HR, hazard ratio; NLR, neutrophil-to-lymphocyte ratio; PLR, platelet-to-lymphocyte ratio. Bold values are significant at $p<0.0$. 
Kitano et al: NLR and PLR in Extrahepatic Cholangiocarcinoma

Table IV. Univariate and multivariate analyses of factors that affect overall survival in patients with extrahepatic cholangiocarcinoma.

\begin{tabular}{|c|c|c|c|c|c|c|}
\hline \multirow[b]{2}{*}{ Variables } & \multicolumn{3}{|c|}{ Univariate analysis } & \multicolumn{3}{|c|}{ Multivariate analysis } \\
\hline & HR & $95 \% \mathrm{CI}$ & $p$-Value & HR & $95 \% \mathrm{CI}$ & $p$-Value \\
\hline Age $>75$ years & 1.14 & $0.63-1.95$ & 0.66 & & & \\
\hline Male gender & 0.74 & $0.46-1.23$ & 0.24 & & & \\
\hline $\mathrm{BMI}>25.0$ & 0.76 & $0.40-1.34$ & 0.35 & & & \\
\hline Reduction of jaundice & 0.94 & $0.55-1.70$ & 0.83 & & & \\
\hline Bilirubin $>10 \mathrm{mg} / \mathrm{dl}$ (1st visit) & 0.99 & $0.54-1.72$ & 0.98 & & & \\
\hline Albumin $<4.0 \mathrm{~g} / \mathrm{dl}$ & 1.64 & $0.95-2.98$ & 0.08 & & & \\
\hline NLR $>2.8$ & 1.19 & $0.67-2.02$ & 0.53 & & & \\
\hline PLR >185 & 1.98 & $1.16-3.27$ & 0.01 & 1.89 & $1.11-3.14$ & 0.02 \\
\hline $\mathrm{CEA}>3.0 \mathrm{ng} / \mathrm{ml}$ & 0.94 & $0.47-1.74$ & 0.86 & & & \\
\hline CA19-9 > 120 U/ml & 1.41 & $0.82-2.35$ & 0.21 & & & \\
\hline Tumor size $>40 \mathrm{~mm}$ & 1.03 & $0.51-1.92$ & 0.92 & & & \\
\hline Surgical time $>720 \mathrm{~min}$ & 1.53 & $0.92-2.49$ & 0.10 & & & \\
\hline Postoperative complication & 1.08 & $0.67-1.79$ & 0.75 & & & \\
\hline Perihilar tumor location & 1.14 & $0.70-1.84$ & 0.59 & & & \\
\hline Poor histologic grade & 0.95 & $0.40-1.96$ & 0.91 & & & \\
\hline $\mathrm{T} 3,4$ & 1.12 & $0.68-1.82$ & 0.65 & & & \\
\hline N1 & 1.89 & $1.14-3.08$ & 0.01 & 1.78 & $1.07-2.93$ & 0.03 \\
\hline $\mathrm{R} 1$ & 1.95 & $1.14-3.24$ & 0.02 & 1.68 & $0.98-2.82$ & 0.06 \\
\hline No adjuvant therapy & 0.99 & $0.60-1.60$ & 0.96 & & & \\
\hline
\end{tabular}

BMI, Body mass index; CI, confidence interval; CA19-9, carbohydrate antigen 19-9; CEA, carcinoembryonic antigen; HR, hazard ratio; NLR, neutrophil-to-lymphocyte ratio; PLR, platelet-to-lymphocyte ratio. Bold values are significant at $p<0.05$.

Table V. Relationship between recurrence sites and NLR/PLR in patients with extrahepatic cholangiocarcinoma.

\begin{tabular}{|c|c|c|c|c|c|c|}
\hline & \multicolumn{3}{|c|}{ NLR } & \multicolumn{3}{|c|}{ PLR } \\
\hline & $\begin{array}{c}\text { High } \\
(n=30)\end{array}$ & $\begin{array}{c}\text { Low } \\
(n=90)\end{array}$ & $p$-Value & $\begin{array}{l}\text { High } \\
(n=30)\end{array}$ & $\begin{array}{c}\text { Low } \\
(n=90)\end{array}$ & $p$-Value \\
\hline Recurrences, n (\%) & $19(63.3 \%)$ & $47(52.2 \%)$ & 0.29 & $20(66.7 \%)$ & $46(51.1 \%)$ & 0.14 \\
\hline \multicolumn{7}{|l|}{ Site of recurrence } \\
\hline Liver & $9(30.0 \%)$ & $22(24.4 \%)$ & 0.55 & $12(40.0 \%)$ & $19(21.1 \%)$ & 0.04 \\
\hline Primary & $8(26.7 \%)$ & $17(18.9 \%)$ & 0.36 & $6(20.0 \%)$ & $19(21.1 \%)$ & 0.90 \\
\hline Lymph node & $2(6.7 \%)$ & $17(18.9 \%)$ & 0.11 & $3(10.0 \%)$ & $16(17.8 \%)$ & 0.31 \\
\hline Lung & $1(3.3 \%)$ & $3(3.3 \%)$ & 1.0 & $1(3.3 \%)$ & $3(3.3 \%)$ & 1.0 \\
\hline Peritoneum & $1(3.3 \%)$ & $10(11.1 \%)$ & 0.20 & $2(6.7 \%)$ & $9(10.0 \%)$ & 0.58 \\
\hline
\end{tabular}

NLR, Neutrophil-to-lymphocyte ratio; PLR, platelet-to-lymphocyte ratio. Bold values are significant at $p<0.05$.

2 van Gulik TM, Kloek JJ, Ruys AT, Busch OR, van Tienhoven GJ, Lameris JS and Gouma DJ: Multidisciplinary management of hilar cholangiocarcinoma (Klatskin tumor): Extended resection is associated with improved survival. Eur J Surg Oncol 37: 65-71, 2011.

3 Nagino M, Ebata T, Yokoyama Y, Igami T, Sugawara G, Takahashi Y and Nimura Y: Evolution of surgical treatment for perihilar cholangiocarcinoma: A single-center 34-year review of 574 consecutive resections. Ann Surg 258: 129-140, 2013.

4 Takahashi Y, Ebata T, Yokoyama Y, Igami T, Sugawara G, Mizuno $\mathrm{T}$ and Nagino M: Surgery for recurrent biliary tract cancer: A single-center experience with 74 consecutive resections. Ann Surg 262: 121-129, 2015.
5 Jarnagin WR, Ruo L, Little SA, Klimstra D, D’Angelica M, DeMatteo RP and Fong Y: Patterns of initial disease recurrence after resection of gallbladder carcinoma and hilar cholangiocarcinoma: Implications for adjuvant therapeutic strategies. Cancer 98: 16891700, 2003.

6 Kobayashi A, Miwa S, Nakata T and Miyagawa S: Disease recurrence patterns after R0 resection of hilar cholangiocarcinoma. Br J Surg 97: 56-64, 2010.

7 McMillan DC, Forrest LM, O'Gorman P, Angerson WJ and McArdle CS: Performance status of male and female advanced cancer patients is independently predicted by mid-upper arm circumference measurement. Nutr Cancer 42: 191-193, 2002. 

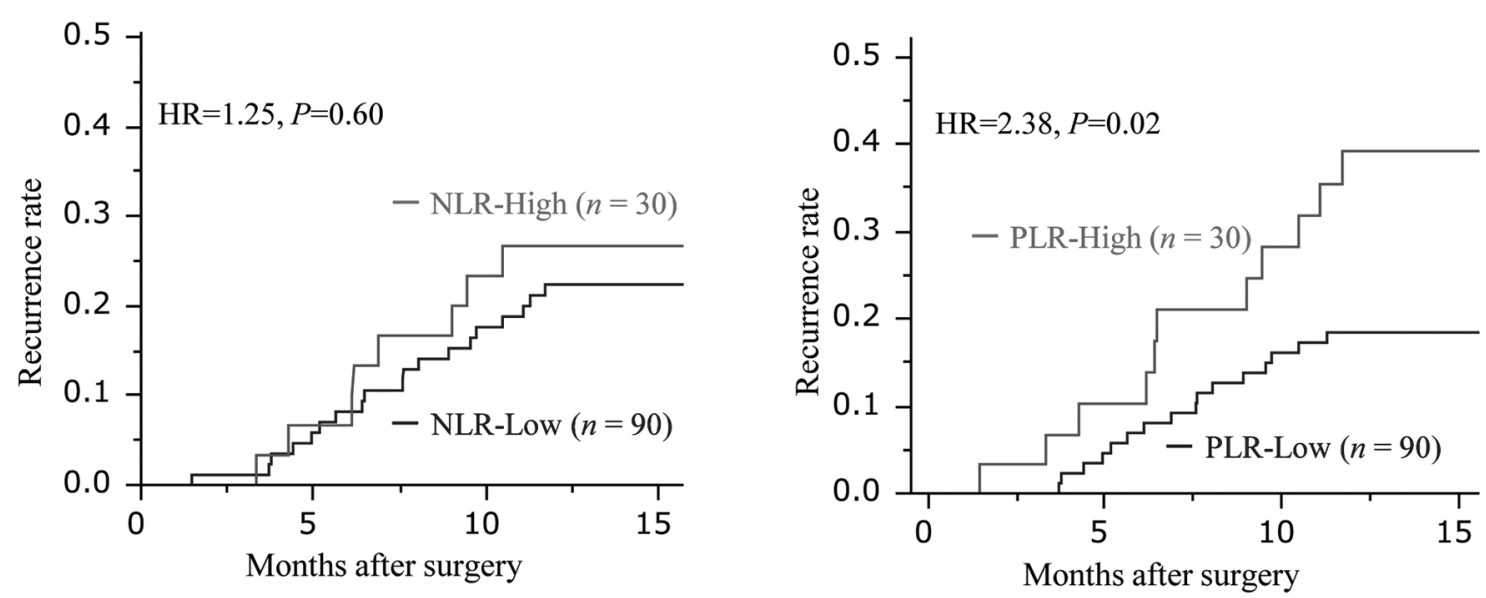

Figure 3. Recurrence rate curves within 1 year by NLR and PLR. NLR, neutrophil-to-lymphocyte ratio; PLR, platelet-to-lymphocyte ratio; HR, hazard ratio.
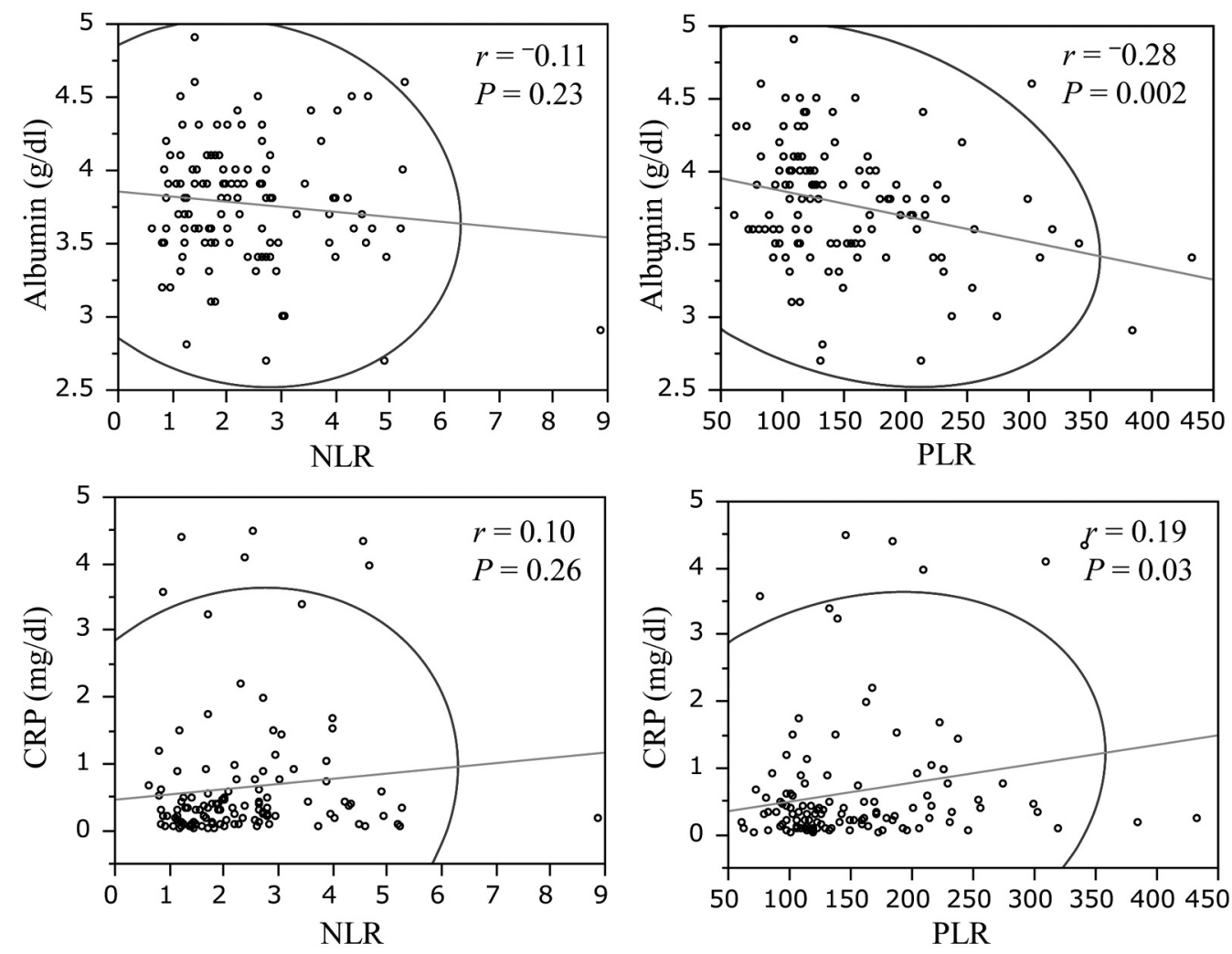

\begin{tabular}{lcccccc}
\hline & NLR-High $(n=30)$ & NLR-Low $(n=90)$ & $P$ & PLR-High $(n=30)$ & PLR-Low $(n=90)$ & $P$ \\
\hline GPS & & & 0.13 & & & 0.01 \\
0 & $17(56.7 \%)$ & $68(75.6 \%)$ & & $15(50.0 \%)$ & $70(77.8 \%)$ \\
1 & $10(33.3 \%)$ & $18(20.0 \%)$ & & $12(40.0 \%)$ & $16(17.8 \%)$ \\
2 & $3(10.0 \%)$ & $4(4.4 \%)$ & & $3(10.0 \%)$ & $4(4.4 \%)$ \\
\hline
\end{tabular}

Figure 4. Relationships between NLR/PLR and other inflammation markers and scores. CRP, C-reactive protein; GPS, Glasgow prognostic score; $N L R$, neutrophil-to-lymphocyte ratio; PLR, platelet-to-lymphocyte ratio. 
8 Forrest LM, McMillan DC, McArdle CS, Angerson WJ and Dunlop DJ: Comparison of an inflammation-based prognostic score (GPS) with performance status (ECOG) in patients receiving platinum-based chemotherapy for inoperable nonsmall-cell lung cancer. Br J Cancer 90: 1704-1706, 2004.

9 Paramanathan A, Saxena A and Morris DL: A systematic review and meta-analysis on the impact of pre-operative neutrophil lymphocyte ratio on long term outcomes after curative intent resection of solid tumours. Surg Oncol 23: 31-39, 2014.

10 Templeton AJ, Ace O, McNamara MG, Al-Mubarak M, VeraBadillo FE, Hermanns T and Amir E: Prognostic role of platelet to lymphocyte ratio in solid tumors: A systematic review and metaanalysis. Cancer Epidemiol Biomarkers Prev 23: 1204-1212, 2014.

11 Kinoshita A, Onoda H, Imai N, Iwaku A, Oishi M, Tanaka K and Matsushima M: The C-reactive protein/albumin ratio, a novel inflammation-based prognostic score, predicts outcomes in patients with hepatocellular carcinoma. Annals of Surgical Oncology 22: 803-810, 2015.

12 Balkwill $\mathrm{F}$ and Mantovani A: Inflammation and cancer: Back to Virchow? Lancet 357: 539-545, 2001.

13 Coussens LM and Werb Z: Inflammation and cancer. Nature 420: 860-867, 2002.

14 Grivennikov SI, Greten FR and Karin M: Immunity, inflammation, and cancer. Cell 140: 883-899, 2010.

15 Miyazaki M, Ohtsuka M, Miyakawa S, Nagino M, Yamamoto $\mathrm{M}$, Kokudo $\mathrm{N}$ and Takada T: Classification of biliary tract cancers established by the Japanese Society of Hepato-BiliaryPancreatic Surgery: 3(rd) English edition. J Hepatobiliary Pancreat Sci 22: 181-196, 2015.

16 Edge SB, Byrd DR, Compton C, Fritz A, Greene FL and Trotti A: American Joint Committee on Cancer (AJCC). Cancer staging manual, 7th ed, New York: Springer-Verlag, 2010.

17 Choi WJ, Cleghorn MC, Jiang H, Jackson TD, Okrainec A and Quereshy FA: Preoperative neutrophil-to-lymphocyte ratio is a better prognostic serum biomarker than platelet-to-lymphocyte ratio in patients undergoing resection for nonmetastatic colorectal cancer. Ann Surg Oncol 22(Suppl 3): S603-S613, 2015.

18 Kim EY, Lee JW, Yoo HM, Park CH and Song KY: The plateletto-lymphocyte ratio versus neutrophil-to-lymphocyte ratio: Which is better as a prognostic factor in gastric cancer? Ann Surg Oncol 22: 4363-4370, 2015.

19 Yodying H, Matsuda A, Miyashita M, Matsumoto S, Sakurazawa N, Yamada $M$ and Uchida E: Prognostic significance of neutrophil-to-lymphocyte ratio and platelet-to-lymphocyte ratio in oncologic outcomes of esophageal cancer: A systematic review and meta-analysis. Ann Surg Oncol 23: 646-654, 2016.

20 Imai T, Koike K, Kubo T, Kikuchi T, Amano Y, Takagi M and Nakahata T: Interleukin-6 supports human megakaryocytic proliferation and differentiation in vitro. Blood 78: 1969-1974, 1991.

21 Klinger MH and Jelkmann W: Role of blood platelets in infection and inflammation. J Interferon Cytokine Res 22: 913-922, 2002.

22 Alexandrakis MG, Passam FH, Moschandrea IA, Christophoridou AV, Pappa CA, Coulocheri SA and Kyriakou DS: Levels of serum cytokines and acute phase proteins in patients with essential and cancer-related thrombocytosis. Am J Clin Oncol 26: 135-140, 2003.

23 Lippitz BE: Cytokine patterns in patients with cancer: A systematic review. Lancet Oncol 14: e218-228, 2013.

24 Bambace NM and Holmes CE: The platelet contribution to cancer progression. J Thromb Haemost 9: 237-249, 2011.
25 Peterson JE, Zurakowski D, Italiano JE Jr., Michel LV, Connors S, Oenick M and Folkman J: VEGF, PF4 and PDGF are elevated in platelets of colorectal cancer patients. Angiogenesis 15: 265$273,2012$.

26 Aoba T, Ebata T, Yokoyama Y, Igami T, Sugawara G, Takahashi $\mathrm{Y}$ and Nagino M: Assessment of nodal status for perihilar cholangiocarcinoma: location, number, or ratio of involved nodes. Ann Surg 257: 718-725, 2013.

27 Kiriyama M, Ebata T, Aoba T, Kaneoka Y, Arai T, Shimizu Y and Nagoya Surgical Oncology G: Prognostic impact of lymph node metastasis in distal cholangiocarcinoma. Br J Surg 102: 399-406, 2015.

28 Hu HJ, Mao H, Shrestha A, Tan YQ, Ma WJ, Yang Q and Li FY: Prognostic factors and long-term outcomes of hilar cholangiocarcinoma: A single-institution experience in China. World J Gastroenterol 22: 2601-2610, 2016.

29 Kang MJ, Jang JY, Chang J, Shin YC, Lee D, Kim HB and Kim SW: Actual long-term survival outcome of 403 consecutive patients with hilar cholangiocarcinoma. World J Surg 40(10): 2451-2459, 2016.

30 Su CH, Tsay SH, Wu CC, Shyr YM, King KL, Lee CH and P'Eng F K: Factors influencing postoperative morbidity, mortality, and survival after resection for hilar cholangiocarcinoma. Ann Surg 223: 384-394, 1996.

31 Konishi M, Iwasaki M, Ochiai A, Hasebe T, Ojima H and Yanagisawa A: Clinical impact of intraoperative histological examination of the ductal resection margin in extrahepatic cholangiocarcinoma. Br J Surg 97: 1363-1368, 2010.

32 Hatzaras I, Schmidt C, Muscarella P, Melvin WS, Ellison EC and Bloomston M: Elevated CA 19-9 portends poor prognosis in patients undergoing resection of biliary malignancies. HPB (Oxford) 12: 134-138, 2010.

33 Kato Y, Takahashi S, Gotohda N and Konishi M: Prognostic impact of the initial postoperative CA19-9 level in patients with extrahepatic bile duct cancer. J Gastrointest Surg 20: 1435-1443, 2016.

34 Horgan AM, Amir E, Walter T and Knox JJ: Adjuvant therapy in the treatment of biliary tract cancer: A systematic review and meta-analysis. J Clin Oncol 30: 1934-1940, 2012.

35 Wang X, Hu J, Cao G, Zhu X, Cui Y, Ji X and Shen L: Phase II study of hepatic arterial infusion chemotherapy with oxaliplatin and 5-fluorouracil for advanced perihilar cholangiocarcinoma. Radiology 7: 160572, 2016.

36 Mano Y, Shirabe K, Yamashita Y, Harimoto N, Tsujita E, Takeishi $\mathrm{K}$ and Maehara Y: Preoperative neutrophil-to-lymphocyte ratio is a predictor of survival after hepatectomy for hepatocellular carcinoma: A retrospective analysis. Ann Surg 258: 301-305, 2013.

37 Shirai Y, Shiba H, Sakamoto T, Horiuchi T, Haruki K, Fujiwara Y and Yanaga K: Preoperative platelet to lymphocyte ratio predicts outcome of patients with pancreatic ductal adenocarcinoma after pancreatic resection. Surgery 158: 360-365, 2015. 